\title{
The Harris-Luck criterion for random lattices
}

\author{
Wolfhard Janke* and Martin Weigel ${ }^{\dagger}$ \\ Institut für Theoretische Physik, Universität Leipzig, Augustusplatz 10/11, 04109 Leipzig, Germany
}

(Dated: November 15, 2018)

\begin{abstract}
The Harris-Luck criterion judges the relevance of (potentially) spatially correlated, quenched disorder induced by, e.g., random bonds, randomly diluted sites or a quasi-periodicity of the lattice, for altering the critical behavior of a coupled matter system. We investigate the applicability of this type of criterion to the case of spin variables coupled to random lattices. Their aptitude to alter critical behavior depends on the degree of spatial correlations present, which is quantified by a wandering exponent. We consider the cases of Poissonian random graphs resulting from the Voronoï-Delaunay construction and of planar, "fat" $\phi^{3}$ Feynman diagrams and precisely determine their wandering exponents. The resulting predictions are compared to various exact and numerical results for the Potts model coupled to these quenched ensembles of random graphs.

PACS numbers: 75.10.Hk, 75.40.Mg, 75.50.Lk
\end{abstract}

\section{INTRODUCTION}

The concept of quenched disorder coupling to the local energy density has evolved to be the main framework for the modeling of the types of randomness found in real physical systems ${ }^{1,2}$. In the presence of frustration this includes the vast field of spin glasses, which in the past decades has attracted an enormous amount of analytical and numerical research, see, e.g., Refs. 2-5. Here, we will be concerned with the simpler case of models with purely ferromagnetic couplings. The first investigations of this problem have considered defects distributed in the system completely at random ${ }^{6,7}$, realized in lattice models, e.g., as random variation of the coupling strengths or random deletion of bonds or lattice sites ${ }^{8-10}$.

The relevance of this random-bond or dilution type of disorder for the universal behavior of spin systems has been the subject of much research ${ }^{8,11-17}$. For the case of models undergoing a continuous phase transition on regular lattices, Harris ${ }^{8}$ argued that for models with a specific-heat exponent $\alpha<0$ the fluctuations in the local transition temperature induced by the disorder degrees of freedom are not strong enough to alter universal features of the model such as the critical exponents. Albeit not originally claimed by $\mathrm{Harris}^{8}$, for the converse case of a positive specific-heat exponent $\alpha$ a significant change of the system's behavior was expected. The precise effect of such a relevant perturbation has been the subject of some debate ${ }^{1,8}$. While it was originally believed that the transition temperature fluctuations might smoothen out the phase transition so far as to completely destroy it, it was later on realized that this, in fact, does not happen and, instead, the system experiences a cross-over from the pure fixed point to a new, disorder fixed point, resulting in a new set of critical exponents and further universal properties such as amplitude ratios ${ }^{9,13,14,18-20}$. This scenario has been especially thoroughly analyzed for the case of the $q=2,3,4$ Potts models in two dimensions, where results from perturbative methods ${ }^{13-15}$ agree well with the outcome of numerical simulation studies ${ }^{10,21-23}$. From this general observation of a smoothening effect of disorder on phase transitions, one might expect that for systems exhibiting a first-order transition in the regular case, disorder of the random-bond type might soften the transition to a continuous one ${ }^{17}$. For the case of two dimensions it could be rigorously established that even an infinitesimally small amount of disorder suffices to indeed induce this behavior ${ }^{24-27}$; in three dimensions, sufficiently strong disorder coupling to the local energy density is numerically found to soften first-order phase transitions to second-order ones, see, e.g., Refs. 28-30.

Obviously, for many physical systems the assumption of an uncorrelated, isotropic distribution of defects is not an adequate description. Instead, due to various reasons the distribution of defects is spatially correlated. This effect occurs isotropically due to long-range interactions between the non-magnetic ions, or in the form of line or higher-dimensional defects ${ }^{31-35}$. In these cases, the reasoning leading to Harris' relevance criterion is no longer directly applicable, but can be generalized accordingly. For the case of algebraically decaying correlations one finds a relevance threshold depending on the dimension of the defects as well as the power of the decay of the correlations ${ }^{31,32}$. For a different model, not covered by the random-bond paradigm, namely the co-ordination number non-periodicity found in quasi-crystals and other aperiodic structures, Luck $^{16}$ formulated a relevance criterion that includes the situations discussed as special cases. There, the "break-even point" for the relevance of randomness in terms of the specific-heat exponent $\alpha$ is shifted from its uncorrelated value $\alpha_{c}=0$ to somewhere in the region $-\infty<\alpha_{c} \leq 1$, depending on the strength of spatial correlations of the disorder degrees of freedom measured by a geometrical fluctuation or wandering exponent. A structure conceptually related to these aperiodic models is given by different varieties of random graphs such as Poissonian Voronoï-Delaunay triangulations ${ }^{36,37}$ or the planar, combinatorial triangulations encountered in the dynamical triangulations approach to quantum gravity resp. the dual planar and orientable $\phi^{3}$ Feynman diagrams ${ }^{38}$. Although spin models on quenched ensembles of these graphs have been considered in numerical 


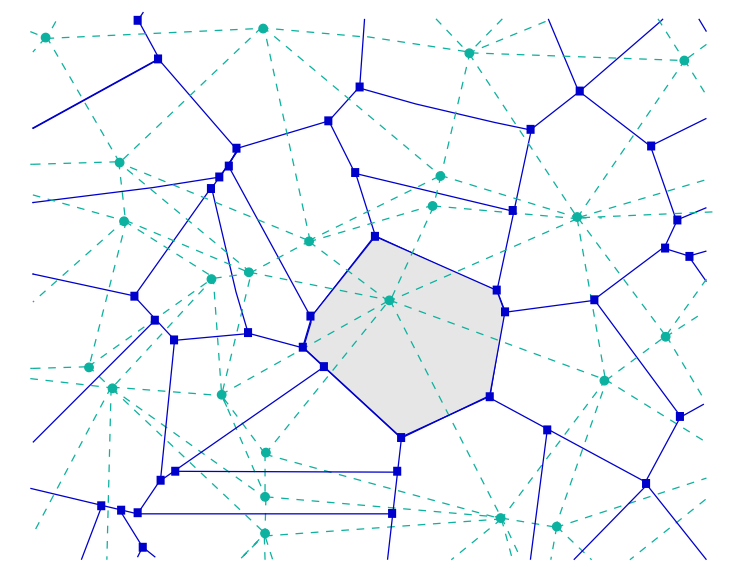

FIG. 1: Patch of a Poissonian Voronoï-Delaunay triangulation of the plane. The spherical, shaded dots denote the randomly distributed generators. The solid lines enclosing these generators define convex polygonal areas whose points are closer to the origin than to any other generator. They form the network of Voronoï cells, which results in a three-valent graph whose vertices are depicted by the dark boxes. Its geometrical dual, consisting of the generators and the connecting dashed lines, is the Delaunay triangulation.

simulation studies ${ }^{39-46}$, no connection has been made as yet with the predictions of Luck $^{16}$ for general systems with connectivity disorder. In particular, the geometrical fluctuation exponents appearing in this relevance criterion have not been determined for the case of these random graphs.

The rest of this paper is organized as follows. In Section II we introduce the ensembles of graphs to be considered, elaborate on their generation in a computer experiment and review some of their known properties. Section III is devoted to a presentation of a version of the Harris-Luck relevance criterion suitable to be applied to the case of random graphs considered here and a discussion of its connection with previous results. In Section IV we introduce different methods to precisely determine the wandering exponent for both graph types and present the results obtained. Finally, Section V contains our conclusions.

\section{RANDOM GRAPHS}

In the following we present construction techniques and properties of two different kinds of two-dimensional topological graphs whose randomness is solely encoded in the degree distribution of their vertices, resulting in a topological connectivity disorder. In contrast to the generic random graphs discussed in the context of scalefree networks, small-world models, etc. ${ }^{47,48}$, the ensembles of random structures considered here are not fully determined by their degree or co-ordination number distributions, but additionally exhibit a well-defined topology and long-range correlations of their disorder degrees (a)

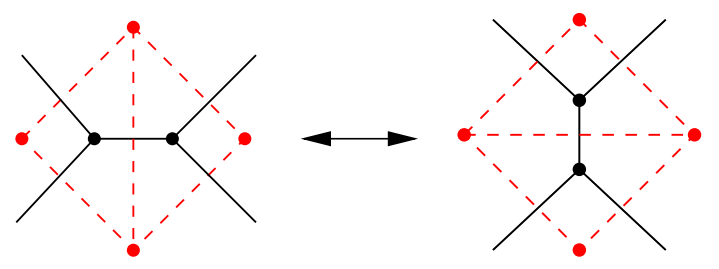

(b)

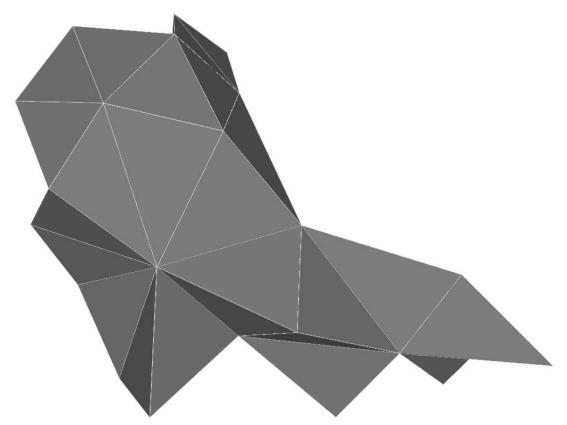

FIG. 2: (a) The link-flip move on two adjacent triangles of a dynamical triangulation (dashed lines). The solid lines denote the corresponding dual, three-valent $\phi^{3}$ graphs. (b) Example of a spherical dynamical triangulation embedded into threedimensional space. Note that the requirement of equilaterality of the triangles is only approximately fulfilled for the embedding shown, since there is no generic exact embedding algorithm for performing it.

of freedom.

\section{A. Poissonian Voronoï-Delaunay Triangulations}

Irregular cell structures or froths appear in a large variety of natural systems, such as foams or biological tissues ${ }^{37}$. The common inverse problem of constructing such a connectivity or cell structure from a given irregular arrangement of vertices (so-called generators) is solved by the Voronoï-Delaunay construction ${ }^{36}$. In two dimensions, the Voronoï cell of a given generator is a convex polygon around it, enclosing the part of its neighborhood which is closer to it than to any other generator, cf. Fig. 1. This is in complete analogy with the notion of a WignerSeitz elementary cell in crystallography. This construction results in the three-valent Voronoï graph and the dual Delaunay triangulation. If the generators are located completely at random, as is the case in the example of Fig. 1, the resulting graph is known as Poissonian Voronoï-Delaunay triangulation ${ }^{36}$.

These lattices are randomly disordered in several respects: edge lengths, cell volumes etc. vary, as well as the co-ordination numbers $q$ of the vertices of the Delaunay triangulation. To facilitate comparison with the second type of random graphs to be introduced below, we here restrict ourselves to the latter aspect of variable coordination numbers, i.e., we do not take any effects from variable lengths or areas into account. Thus, the distri- 
bution $P(q)$ of co-ordination numbers is the only random variable involved. Additionally, to eliminate surface effects, the generators are randomly placed on the surface of a sphere instead of a patch of the plane. Thus, the resulting random graphs are triangulations of spherical topology.

From the Euler relations, the average co-ordination number is a topological invariant for a fixed number of triangles in two dimensions, given by ${ }^{38}$

$$
\bar{q}=\frac{1}{N} \sum_{i} q_{i}=6 \frac{N}{N+4}
$$

for any closed, spherical triangulation, where $N$ denotes the number of triangles. In the limit of infinite triangulations, $N \rightarrow \infty$, one thus obviously has $\langle\bar{q}\rangle=\left\langle q_{i}\right\rangle=6$. The second moment of $q$ is not exactly known, but is numerically found to be $\mathrm{e}^{36,49}$

$$
\mu_{2} \equiv\left\langle q_{i}^{2}\right\rangle-\left\langle q_{i}\right\rangle^{2} \approx 1.781,
$$

as $N \rightarrow \infty$. It turns out that the random variables $q_{i}$ are not independently distributed, but are reflecting a spatial correlation of the disorder degrees of freedom in addition to the trivial correlation induced by the constraint (1). The form of these correlations for nearest-neighbor vertices is commonly described by the Aboav-Weaire law ${ }^{36}$, which states that the total expected number of edges of the neighbors of a $q$-sided cell, $q m(q)$, should vary linearly with $q$,

$$
q m(q)=(6-a) q+b,
$$

where $a$ and $b$ are some parameters. In turns out, however, that Eq. (3), albeit being a good effective description for a large variety of cell systems including the case of Poissonian Voronoï-Delaunay triangulations, is merely a leading-order and not an exact property of these systems $^{37}$.

\section{B. Dynamical Triangulations}

An ensemble of planar triangulations with properties very different from those of the Poissonian VoronoïDelaunay lattices is given by the so-called dynamical triangulations model, which has been used as a constructive approach to discrete Euclidean quantum gravity in two dimensions ${ }^{38}$. This ensemble of combinatorial triangulations is defined as that of all gluings of equilateral triangles to closed surfaces of a given topology (such as, e.g., that of a sphere), where all gluings are counted with equal probability. A dynamical way of generating such graphs is the repeated application of so-called linkflip moves which re-wire a given network [see Fig. 2(a)]. It is known that this procedure converges to a limiting distribution with specific properties described below. Since this flip dynamics introduces large temporal correlations between subsequent random triangulations, we

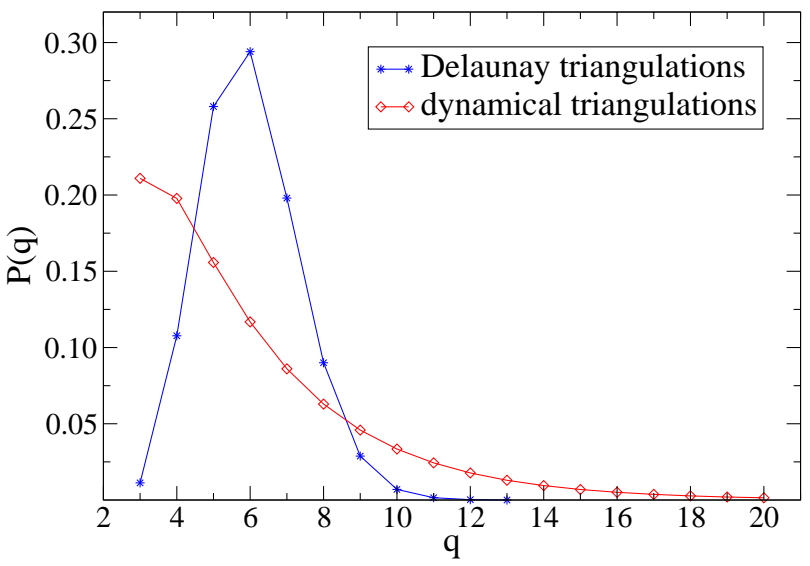

FIG. 3: Comparison of the degree distributions $P(q)$ of Poissonian Delaunay triangulations and dynamical triangulations in the limit of an infinite number of triangles. The results are taken from Refs. 49,50.

do not actually use it to generate the graph instances needed below, but instead revert to a recursive insertion technique known to yield the same graph distribution, but independent graph realizations in each step ${ }^{51}$. In this construction, no edge length differences are involved and, again, the randomness is solely encoded in the degree distribution $P(q)$ of the vertices. Although these objects are entirely defined in terms of their intrinsic connectivity properties, an embedding of an example dynamical triangulation into three-dimensional space is shown for illustration purposes in Fig. 2(b). Technically, the geometrical duals of these triangulations are given by the ensemble of planar, "fat" (i.e., orientable) $\phi^{3}$ Feynman diagrams without tadpoles and self-energy insertions. The statistics of these objects can be explicitly treated by means of matrix models ${ }^{52,53}$, leading to exact solutions for the co-ordination number distribution $P(q)$ and many other properties of the model ${ }^{38,50}$. Compared to the Poissonian Voronoï-Delaunay model, fluctuations are much more pronounced for these graphs, and it can be shown that the variance of co-ordination numbers approaches ${ }^{50,54}$

$$
\mu_{2}=21 / 2
$$

as $N \rightarrow \infty$, whereas Eq. (1) still holds. Also, the AboavWeaire law (3) correctly describes the leading-order behavior of the nearest-neighbor correlations ${ }^{54}$.

Considering more geometric properties of the graphs, the differences between both ensembles become even more pronounced. Especially, the quantum gravity graphs of the dynamical triangulations model can be shown to be highly fractal, being self-similarly composed of "baby universes" branching off from the main surface, i.e., macroscopical subgraphs attached to the main body by only a few links ${ }^{55}$. This fractal structure leads to an exceptionally large internal Hausdorff dimension 
of $d_{h}=4$ as compared to the topological dimension of two ${ }^{56,57}$, whereas for the Poissonian Voronoï-Delaunay triangulations the Hausdorff dimension remains at the trivial value of the topological dimension. The differences between the two graph ensembles are also very strikingly seen in the distributions $P(q)$ of co-ordination numbers as depicted in Fig. 3. While $P(q)$ is peaked at $q=6$ for the Delaunay triangulations, it drops monotonically starting from $q=3$ for the case of dynamical triangulations, and large co-ordination numbers are much more probable for the latter lattice type. It can be shown that for large co-ordination numbers the distribution $P(q)$ falls off as $\exp (-\sigma q \ln q)$ with $\sigma \approx 2$ for Poissonian random lattices ${ }^{49}$, whereas for dynamical triangulations it declines much slower proportional to $\exp (-\sigma q)$ with $\sigma=\ln (4 / 3) \approx 0.3$ (Ref. 50).

\section{THE RELEVANCE CRITERION}

Trying to decide for which kind of models disorder of the random-bond type constitutes a relevant perturbation, Harris ${ }^{8}$ suggested the following line of reasoning. For a system of uncorrelated random bonds, the fluctuations $\sigma_{R}(J)$ of the average coupling $J$ in patches of the lattice decline with the linear patch size $R$ according to the central limit theorem, that is,

$$
\sigma_{R}(J) \sim R^{-d / 2},
$$

where $d$ denotes the spatial dimension of the system. Since disorder of the random-bond type couples to the local energy density, local fluctuations of the coupling induce such fluctuations of the effective local transition temperature, which decline identically with increasing patch size. Approaching the critical point $t \equiv(T-$ $\left.T_{c}\right) / T_{c}=0$, the fluctuations in a correlation volume scale as

$$
\sigma_{\xi}(J) \sim \xi^{-d / 2} \sim t^{\nu d / 2},
$$

where the power-law divergence $\xi \sim t^{-\nu}$ of the correlation length was used. For the critical behavior of the pure system to persist, these fluctuations should die out as the reduced temperature is linearly tuned to the critical point $t=0$, i.e., one should have $\nu d / 2>1$ or, with appeal to hyper-scaling, $\alpha<0$. In the converse case $\alpha>0$ an altered universal behavior might me expected. Later on, Chayes et al. ${ }^{11,12}$ showed that under quite general conditions the specific-heat exponent of the disordered system, $\alpha_{\text {dis }}$, should be negative. This seemed plausible, since $\alpha$ was believed to coincide with the crossover or stability exponent of the respective renormalization-group (RG) fixed point ${ }^{58,59}$. Later on, however, it was claimed that in some cases the stability exponent might differ from $\alpha$, such that even for positive $\alpha$ the regular critical behavior would prevail ${ }^{60}$. Several examples of such behavior, and even the opposite case of disorder being a relevant perturbation albeit $\alpha<0$, have been explicitly constructed for the case of hierarchical lattices ${ }^{61-65}$. Thus, the general validity of the relevance criterion implied by Harris' argument has recently been the object of some debate, see, e.g., Refs. 66-68.

As soon as the assumption of uncorrelated disorder degrees of freedom is relaxed, Harris' reasoning is no longer applicable as it stands. For a random-bond model with long-range correlations of the bond variables, Weinrib and Halperin ${ }^{31}$ performed a renormalization group calculation, which was later on refined ${ }^{32}$. They find that, if the disorder degrees of freedom are correlated algebraically with a decay exponent $a<d$, disorder is irrelevant if $\alpha<2-2 d / a$, whereas in the opposite case, the system flows towards a new, long-range correlated disorder fixed point. For more general disorder degrees of freedom and types of correlations including the ensembles of random graphs considered here, this argument can be adapted in the spirit of Luck's reasoning for the case of aperiodic structures ${ }^{16}$ as follows. Consider a patch $P$ of spherical shape with radius $R$ and a volume of $B(R)$ vertices $^{82}$ on a given realization of a triangulation. The average co-ordination number in $P$,

$$
J(R) \equiv \frac{1}{B(R)} \sum_{i \in P} q_{i},
$$

fluctuates around its expected value $J_{0}=\bar{q}$ [cf. Eq. (1)]. As the size of the patch is increased, $R \rightarrow \infty$, these fluctuations decay as

$\sigma_{R}(J) \equiv\left\langle\left|J(R)-J_{0}\right|\right\rangle / J_{0} \sim\langle B(R)\rangle^{-(1-\omega)} \sim R^{-d_{h}(1-\omega)}$,

defining the wandering exponent $\omega$ of the considered graph type. The Hausdorff dimension $d_{h}$ enters here to account for the cases of fractal graphs. In Eq. (8), the averages $\langle\cdot\rangle$ are to be understood as the ensemble averages of the considered class of graphs of a given total size. While for $\omega=1 / 2$ the usual $1 / \sqrt{\langle B(R)\rangle}$ behavior of uncorrelated random variables is recovered, for random lattices with long-range correlations of the co-ordination numbers one expects $\omega>1 / 2$, leading to a slowed-down decay of fluctuations. Near criticality, the fluctuation $\sigma_{\xi}(J)$ of the average co-ordination number in a correlation volume induces a local shift of the transition temperature proportional to $|t|^{d_{h} \nu(1-\omega)} \mu_{2}^{1 / 2}$. For the regular critical behavior to persist, these fluctuations should die out as the critical point $t=0$ is approached. This is the case when $\omega$ does not exceed the threshold value

$$
\omega_{c}=1-\frac{1}{d_{h} \nu}=\frac{1-\alpha}{2-\alpha},
$$

provided that hyper-scaling is in effect. Conversely, for fluctuations satisfying $\omega>\omega_{c}$ a new type of critical behavior could occur. Re-writing Eq. (9) as

$$
\alpha_{c}=\frac{1-2 \omega}{1-\omega},
$$

it is obvious that for $\omega=1 / 2$ the Harris criterion is recovered. 
It is easily seen that the case of algebraically decaying correlations discussed in Ref. 31 is included in Eq. (10) as a special case. Specifically, for the case of connectivity disorder considered here, an isotropic power-law correlation would be given by

$$
G_{q q}(i, j) \equiv\left\langle\delta q_{i} \delta q_{j}\right\rangle \sim \operatorname{dist}(i, j)^{-a},
$$

where $\delta q_{i}=q_{i}-\bar{q}$ denotes the co-ordination number defect at vertex $i$ and the distance $\operatorname{dist}(i, j)$ is defined as the unique number of links in the shortest path of links connecting the two vertices $i$ and $j$. Then, the fluctuation of the mean $\overline{\delta q_{R}}$ over a spherical patch $P$ of radius $R$ is given by

$$
\begin{aligned}
\sigma^{2}\left(\overline{\delta q_{R}}\right) & =\frac{\sigma^{2}(\delta q)}{R^{d_{h}}}+\frac{1}{R^{2 d_{h}}} \sum_{i \neq j \in P}\left\langle\delta q_{i} \delta q_{j}\right\rangle \\
& \sim \text { const } \times R^{-d_{h}}+\text { const } \times R^{-a}
\end{aligned}
$$

as $R \rightarrow \infty$. For short-range correlations $a \geq d_{h}$ the leading behavior is that of Eq. (5), such that the Harris criterion stays in effect. For long-range correlations $a<$ $d_{h}$, on the other hand, the leading term is proportional to $R^{-a}$. Comparing to Eq. (8), we arrive at the following expression,

$$
\omega=1-a / 2 d_{h},
$$

such that from Eq. (10) we arrive at $\alpha_{c}=2-2 d_{h} / a$, in agreement with the direct observation in Ref. 31.

Since for systems with sufficiently long-range correlations of the disorder degrees of freedom $\omega>1 / 2$, such correlated disorder is more relevant than uncorrelated randomness in the sense that a change of universality class can already be expected for some range of negative values of $\alpha$, cf. Eq. (10). If, on the other hand, correlations decay exponentially, the threshold $\alpha_{c}=0$ of the Harris criterion should stay in effect.

\section{CORRELATORS AND WANDERING EXPONENTS}

In view of the discussion presented in Sec. III, two complementary approaches towards a numerical determination of the wandering exponent $\omega$ present themselves, either a direct evaluation of the scaling of the fluctuations defined in Eq. (8) or an analysis of the correlation function $G_{q q}(i, j)$ of the disorder degrees of freedom defined in Eq. (11) to infer an estimate of $\omega$ via Eq. (13). Both of these methods will be applied for the cases of Poissonian Voronoï-Delaunay as well as dynamical triangulations. For both graph ensembles, a quenched average has to be taken over a number of graph realizations of the considered ensemble. For this purpose, we generate lattices of spherical topology and consider the triangulations of varying co-ordination numbers as the basic objects and refer to their geometric duals as the "dual" lattices.

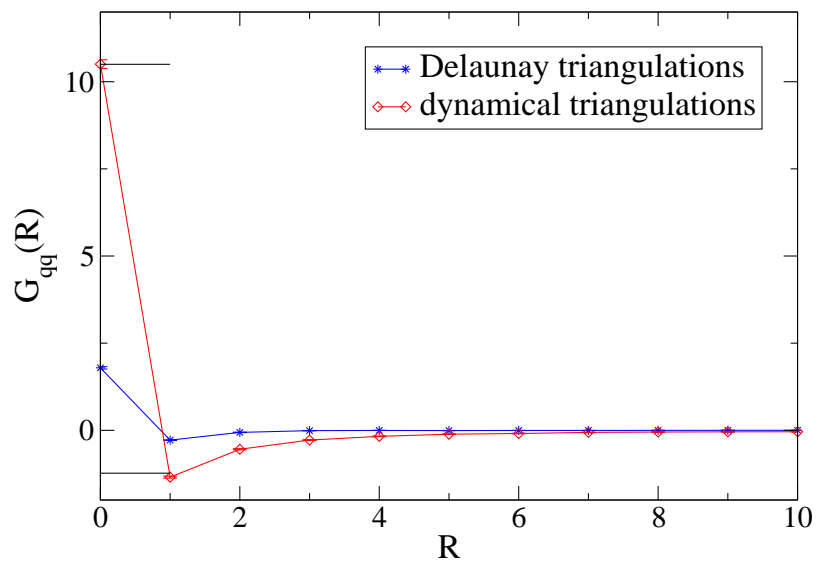

FIG. 4: Comparison of the connected correlator $G_{q q}(R)$ of the co-ordination number defects $\delta q=q_{i}-\bar{q}$ of Poissonian Delaunay triangulations and dynamical triangulations of size $N=125000$ triangles. The connecting lines are only drawn to guide the eye. The two short horizontal lines indicate the exact result $\mu_{2}=10.5$ (cf. Ref. 54) and the value $G_{q q}(1) \approx$ -1.2295 for the dynamical triangulations discussed in App. A, both valid in the limit $N \rightarrow \infty$.

\section{A. Analysis of Correlation Functions}

For the numerical determination of the correlator $G_{q q}(i, j)$ a decomposition of the graphs into spherical shells around a given vertex is performed. This is done by first picking a vertex of the triangulation at random and a subsequent slicing of the graph into shells of equal geodesic link-distance around that vertex. For that given choice of initial vertex $i_{0}$, two fundamental observables can be measured, the volumes of the decomposition shells,

$$
g_{11}^{i_{0}}(R)=\sum_{\operatorname{dist}\left(i, i_{0}\right)=R} 1
$$

which, if properly averaged over, give the correlator of the unit operator, and,

$$
g_{q q}^{i_{0}}(R)=\sum_{\operatorname{dist}\left(i, i_{0}\right)=R} \delta q_{i_{0}} \delta q_{i}
$$

the average of which gives the correlator of Eq. (11). In view of Eq. (7) it is obvious that $B(R)=\sum_{r=0}^{R} g_{11}^{i_{0}}(r)$. In the context of the dynamical triangulations approach to quantum gravity, there has been some debate on how to properly define (connected) correlators on an ensemble of random graphs, see, e.g., Ref. 69. The uncertainty concerns the order of taking the averages over a single graph and the graph ensemble, which has not been explicitly specified in Eq. (11). The two possibilities are given by averaging the expressions of Eqs. (14) and (15) individually, i.e.,

$$
G_{q q}(R)=\frac{\left\langle g_{q q}^{i_{0}}(R)\right\rangle}{\left\langle g_{11}^{i_{0}}(R)\right\rangle}
$$




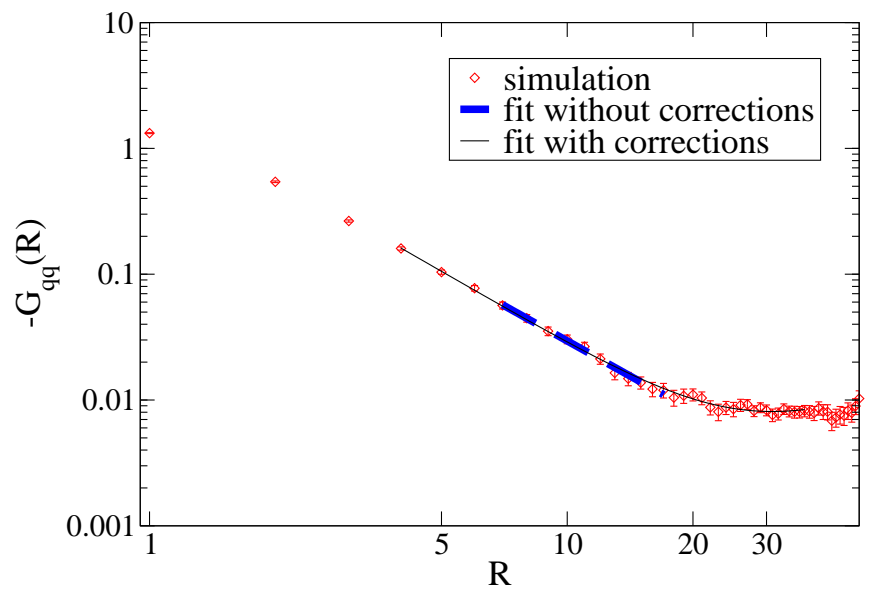

FIG. 5: Decay of the correlator $-G_{q q}(R)$ of the co-ordination numbers of dynamical triangulations with $N=500000$ triangles in a double logarithmic plot. The lines show fits of the functional form (18) to the data, where either the correction term was omitted ( $B=0$, thick dashed line) or included with $B$ and $b$ as free parameters (solid line). The ranges of the lines indicate the range of data points included in the fits.

where the average $\langle\cdot\rangle$ denotes a combined average over the starting vertices $i_{0}$ and the graph realizations under consideration, which is obviously equivalent to the ensemble average of the graphs. The additional average over the starting vertices $i_{0}$ is merely included to improve the statistical accuracy in practical applications. On the other hand, one could also average on the level of the fraction,

$$
G_{q q}(R)=\left\langle\frac{g_{q q}^{i_{0}}(R)}{g_{11}^{i_{0}}(R)}\right\rangle .
$$

It turns out that, at least for the quantum gravity graphs, these two ways of performing the average yield strikingly different results, even in the thermodynamic limit ${ }^{69}$. The main motivation for using Eq. (16) is that an expression of the form $\left\langle g_{\phi \phi}^{i_{0}}(R)\right\rangle$, if integrated over all distances $R$, still yields some kind of susceptibility of the associated operator $\phi$. Otherwise, however, correlators defined according to Eq. (16) behave rather pathologically. Definition (17), on the other hand, corresponds to the natural probabilistic definition of the average correlation of a given quantity at distance $R$, and is thus the unique "correct" definition in the given context and will be used throughout. Note, that in this case the average has to be performed carefully, since the maximum linear separation $R_{\max }$ of two points on the graph is not universal, but depends on the graph realization as well as on the chosen initial vertex.

Figure 4 shows an overview of the short-distance behavior of the correlator $G_{q q}(R)$ defined according to Eq. (17) for Poissonian Delaunay as well as for dynamical triangulations as measured by averaging over 100 graph realizations sampled with one different starting vertex per

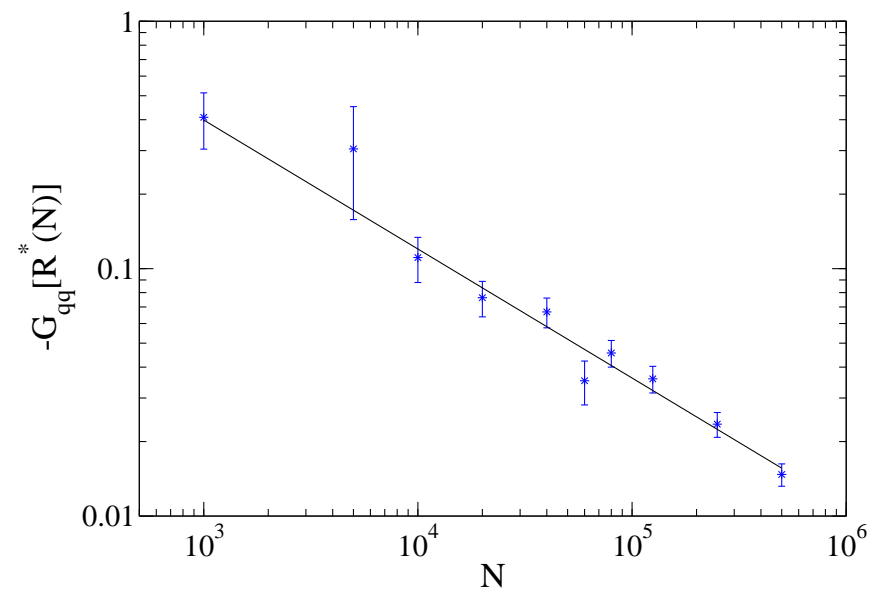

FIG. 6: Finite-size scaling of the co-ordination number correlator $-G_{q q}\left[R^{*}(N)\right]$ at distances $R^{*}(N)$ according to Eq. (21) for dynamical triangulations of sizes ranging from $N=1000$ to $N=500000$ in a $\log$-log plot. The solid line shows a fit of the functional form (20) to the data.

1000 graph vertices each. The statistical errors here and in the following were determined via jackknifing (see, e.g., Refs. 70,71) over the 100 different graph realizations. Obviously, $G_{q q}(0)=\mu_{2}$, corresponding to the values cited above in Sec. II. Note, that the negative correlation at distances $R>0$ reflects the fact, expressed in the AboavWeaire law (3), that a vertex with co-ordination number $q>6$ tends to have neighbors with $q<6$ and vice versa. The correlation for nearest-neighbor points for the case of dynamical triangulations can be calculated in the thermodynamic limit by a series expansion of the results found in Ref. 54, yielding $G_{q q}(1) \approx-1.2295$, cf. App. A. This result is in reasonable agreement with the value found numerically for the example of $N=125000$ triangles presented in Fig. $4, G_{q q}(1) \approx-1.34$. The remaining difference gives a first indication of the presence of large finite-size corrections for the case of the highly fractal quantum gravity graphs. Going beyond $R=1$, a short glance at Fig. 4 reveals that the correlations are much more long-ranged for the case of the quantum gravity graphs than for the Voronoï-Delaunay random lattices. In fact, for the Delaunay triangulations we find that for the graph sizes up to $N=500000$ triangles considered, co-ordination numbers of vertices at distances $R \gtrsim 10$ apart are effectively uncorrelated up to the precision of our calculations. Due to this smallness of correlations it is not possible to determine their exact functional form from the accuracy of our measurements. However, a simple exponential decay can be fitted reasonably well to the behavior found. Additional checks in support of this picture will be presented below in this Section and in Sec. IV B.

For the case of the quantum gravity graphs, we determine the asymptotic behavior of the correlator (17) by fitting a suitably parametrized function to the numerical data. For the algebraic decay expected for this graph 
TABLE I: Results of fits of the functional form (18) to the correlator $G_{q q}(R)$ for dynamical triangulations with $N=500000$ triangles and with various restrictions.

\begin{tabular}{cccccc}
\hline \hline Restriction & $A$ & $a$ & $B$ & $b$ & $R_{\min }-R_{\max }$ \\
\hline$B=0$ & $-2.11(45)$ & $1.854(105)$ & 0 & - & $7-17$ \\
$b=3$ & $-2.37(29)$ & $1.938(92)$ & $5.6(19) \times 10^{-5}$ & 3 & $4-37$ \\
$b=4$ & $-2.04(21)$ & $1.841(75)$ & $1.27(40) \times 10^{-6}$ & 4 & 0.981 \\
none & $-2.30(24)$ & $1.919(76)$ & $3.1(67) \times 10^{-5}$ & $3.15(57)$ & $4-37$ \\
\hline \hline
\end{tabular}

type, we make the ansatz,

$$
G_{q q}(R)=A R^{-a}\left(1+B R^{b}\right)
$$

for $R \geq 1$, taking into account an additional effective scaling correction with exponent $b$. From the discussion in App. B we expect $b=d_{h}=4$ to be a reasonable choice as long as no additional, more relevant, non-analytic corrections are present ${ }^{83}$. Note that, since this "correction" term $R^{b}$ is more singular than the leading term $R^{-a}$, this form is merely an effective description for a finite graph and distances small compared to the linear extent of the graph. Figure 5 depicts the behavior of the correlator of co-ordination numbers for the case of quantum gravity graphs as well as fits of the form (18) to the data. We expect the range of applicability of the form (18) to be limited at both sides: for very small distances $R$, discretization effects are observed, whereas for large distances finite-size effects modify the expected behavior, i.e., higher-order terms neglected in Eq. (18). To account for these limitations, sampled points from both sides of the $R$ range are successively dropped from the fit while monitoring the goodness-of-fit parameters $\chi^{2}$ resp. $Q .{ }^{84}$ We find restricted fits with the correction exponent $b$ fixed at values $b=4$ or, alternatively, $b=3$, to match the data reasonably well. A full four-parameter fit with variable exponent $b$ yields the intermediate value $b=3.15(57)$, in total indicating the presence of higherorder resp. non-analytic corrections. The fit results are compiled in Table I. Since we consider the correction term of Eq. (18) as an effective description, we take the fit with a variable value of the correction exponent $b$ as the most reliable and quote as our best estimate of the decay exponent from this method

$$
a=1.919(76) \text {. }
$$

Due to the cross-correlations of $G_{q q}(R)$ for different distances $R$, the errors estimated by standard fit routines are biased; thus, instead, the errors were estimated by jackknifing over the whole fitting procedure. As a check of whether the used number of replicas in the disorder average is sufficient, we also performed the same analysis with subsets of the 100 realizations. Apart from the effect of rather sudden jumps of the fits into different, nearby minima, which always tend to occur with non-linear fitting procedures, we find all results completely consistent with each other within statistical errors. Thus, e.g., for the unrestricted fit of the form (18) we find (using the same ranges $R_{\min }-R_{\max }$ as before) $a=2.032(115)$ using only 50 graphs and $a=1.885(216)$ using only 10 graphs.

A different way to determine the decay exponent $a$ is based on a direct application of the finite-size scaling (FSS) behavior of the correlator. From FSS arguments it is known that the value of the correlator at a distance $R^{*}(N)$ scaled linearly with the size of the system behaves as

$$
G_{q q}\left[R^{*}(N)\right] \sim N^{-a / d_{h}}
$$

cf. App. B. The position of the reference points $R^{*}$ should be selected well within the scaling region of the considered graph size. Here, we take the largest used graph size as a reference and choose

$$
R^{*}(N)=15\left(\frac{N}{5 \times 10^{5}}\right)^{1 / d_{h}}
$$

where $d_{h}=4$. Obviously, the resulting distances $R^{*}(N)$ will in general be fractional numbers, for which no data are directly available. Since the accessible linear graph sizes $N^{1 / d_{h}}$ are rather small, this discretization effect is quite pronounced. To circumvent this problem, we take a fit of the functional form (18) to the correlator with the correction term included as a suitable interpolation of the data used to estimate the value of $G_{q q}\left[R^{*}(N)\right]$. As an estimate of the statistical error, we take the error computed for the closest integer distance. Figure 6 shows the resulting scaling plot and a fit of the functional form (20) to the data. We find the data not precise enough to resolve any corrections to the expected scaling form. The fit, including a series of graph sizes ranging from $N=1000$ to $N=500000$, yields an estimate for the decay exponent of

$$
a=2.09(13)
$$

with quality $Q=0.53$. For completeness, we have also performed the same analysis for the case of random Delaunay triangulations. As expected, the resulting values of $G_{q q}\left[R^{*}(N)\right]$ do not show scaling according to Eq. (20), backing up our conjecture that the correlations are not algebraic in this case. 


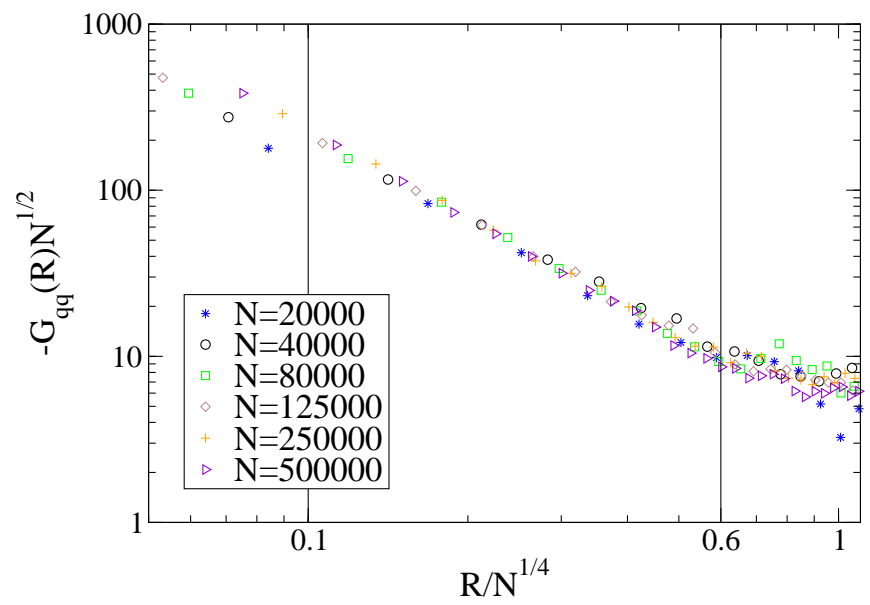

FIG. 7: Scaling collapse on the universal scaling function $\hat{W}$ defined in Eq. (B2) of the co-ordination number correlator $G_{q q}(R)$ for dynamical triangulations of sizes $N=20000$ to $N=500000$ triangles. The vertical lines indicate the extent of the scaling window.

Exploiting the FSS form (B2) of the correlator, it is also possible to re-scale the data such as to model the universal FSS function $\hat{W}$ introduced in Eq. (B2). Thus, plotting $G_{q q}(R) N^{a / d_{h}}$ as a function of the reduced distance $R / N^{1 / d_{h}}$ should, within the scaling region, produce data lying on a single master curve, irrespective of the lattice volume $N$ under consideration. Such a scaling plot is shown in Fig. 7, where a nice scaling collapse of the data for an intermediate range of reduced distances $R / N^{1 / d_{h}}$ is observed. For producing this scaling plot, we assumed $a=2$, in agreement with the results found so far and further evidence to be presented below. The quality of the collapse does not change visibly on slightly changing the value of the exponent $a$ to one of the values $a=1.919$ or $a=2.09$ found before. The extension of the scaling window in reduced distance $R / N^{1 / d_{h}}$ can be very nicely read off from Fig. 7 to be

$$
0.1 \lesssim \frac{R}{N^{1 / d_{h}}} \lesssim 0.6
$$

yielding, e.g., $1 \leq R \leq 7$ for $N=20000$ and $3 \leq R \leq 16$ for $N=500000$, in perfect agreement with the observations from the direct analysis of the correlator.

Extending the analogy between the magnetic correlation function in a critical spin system and the correlator considered here, one notes that the decay exponent $a$ defined above is related to the conventional critical exponents by $a=2-d_{h}-\eta$. A convenient and very precise method of determining $\eta$ is to consider the scaling of the integrated correlation function corresponding to the susceptibility instead of analyzing the correlation function directly. The associated critical exponent $\gamma / \nu$ then allows one to infer $\eta$ via the scaling relation $\eta=2-\gamma / \nu$. Unfortunately, however, it follows from the relation (1) that the average co-ordination number on a closed triangulation is a fixed number, depending only on the number

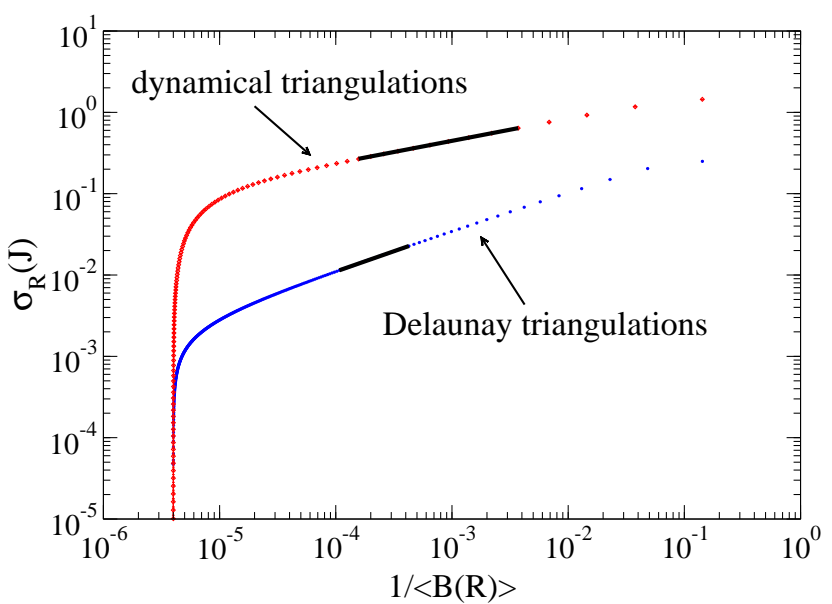

FIG. 8: Decay of the averaged fluctuation $\sigma_{R}(J)$ of coordination numbers against the inverse averaged integrated shell volume $1 /\langle B(R)\rangle$ according to Eqs. (7) and (8) for dynamical triangulations (upper points) and Poissonian Delaunay triangulations (lower points). For both ensembles lattices with $N=500000$ triangles have been used. The solid lines show fits of the functional form (8) to the data.

of triangles $N$. Thus the corresponding susceptibility always vanishes identically, such that the method outlined above cannot be applied here.

\section{B. Averaged Fluctuations}

Instead of an analysis of the correlator of co-ordination numbers, the wandering exponent $\omega$ can be directly estimated by considering the scaling of the averaged fluctuations of co-ordination numbers and recurring to relation (8). This approach has the advantage of giving a numerical result also for the case of correlations decaying other than algebraically, as we saw for the Poissonian Voronoï-Delaunay random lattices. We define the average fluctuation $J(R)$ as indicated in Eq. (7) with the average performed on the level of the fraction, in complete analogy with the definition (17) of the correlator. As before, the quenched average is performed over one starting vertex per 1000 vertices of the graph as well as over 100 different graph realizations for each graph size. Figure 8 shows the resulting decay of $\sigma_{R}(J)$ against the inverse averaged integrated shell volume $1 /\langle B(R)\rangle$. The smaller number of data points for the case of dynamical triangulations results from the smaller effective linear extent $\sim N^{1 / d_{h}}$ due to their large fractal dimension. The relation of the two data sets nicely illustrates the much stronger correlations present in the dynamical triangulations model. From Eq. (8), we expect a linear decline of the curve in a logarithmic presentation, the slope being given by $1-\omega$. On the other hand, corrections to the conjectured scaling behavior for very small distances $R$ due to discretization effects as well as for large distances comparable to the effective linear extent of the 


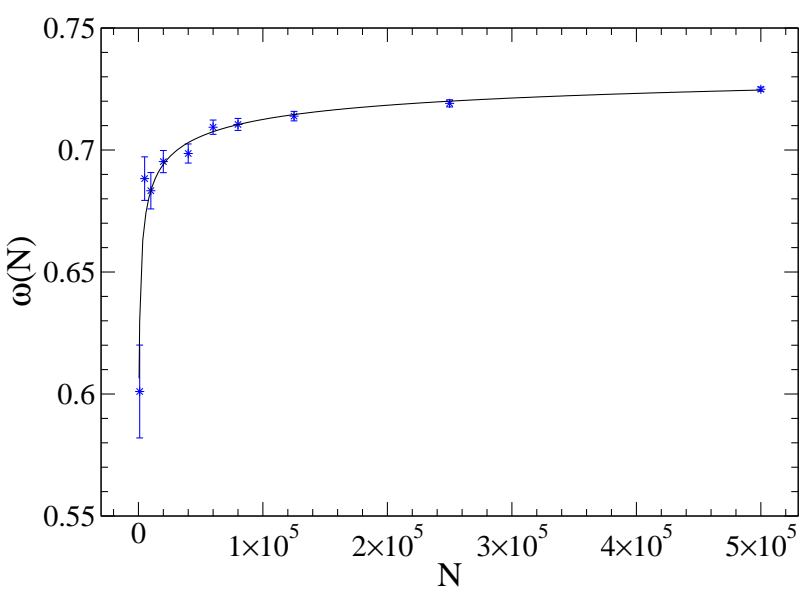

FIG. 9: Finite-size scaling of the estimates of the wandering exponent $\omega(N)$ from fits of the functional form (8) to the data for dynamical triangulations of sizes $N=1000$ up to $N=500000$ triangles. The line shows a fit of the form (25) to the data.

lattices have to be taken into account. In Fig. 8 the scaling window of algebraic behavior according to Eq. (8) is nicely visible. The dramatic, exponential drop of the fluctuations as $1 /\langle B(R)\rangle$ approaches ${ }^{85} 1 /(2+N / 2)$ is an effect of the topological constraint (1), as a consequence of which the fluctuation $\sigma_{R}(J)$ vanishes identically at the maximum observed distance $R=R_{\max }$. To obtain reliable estimates for the wandering exponent $\omega$ from a fit of the functional form (8) to the data, we again successively drop points from either side of the interval of distances $R$ while monitoring the goodness-of-fit parameters $\chi^{2}$ resp. $Q .{ }^{84}$ Note that, as before, due to the cross-correlations between the values of $\sigma_{R}(J)$ for neighboring distances $R$, we have to resort to jackknifing over the whole fitting procedure to arrive at reliable error estimates.

From the fits to the data for the maximum graph sizes $N=500000$ depicted in Fig. 8, we arrive at the following estimates for $\omega$,

$$
\omega= \begin{cases}0.50096(55), & R=21, \ldots, 41, \text { Delaunay tr. } \\ 0.72492(86), & R=5, \ldots, 14, \quad \text { dynamical tr.. }\end{cases}
$$

From the experience with the analysis of the correlator presented above in Sec. IV A, we expect rather pronounced finite-size effects still to be present at least for the case of the dynamical triangulations, such that the quoted statistical error certainly does not account for all of the deviation from the asymptotic result. Note that the result for Delaunay triangulations is perfectly consistent with a wandering exponent $\omega=1 / 2$ resulting from either power-law correlations with an exponent $a>2$, which, however, did not show up in the analysis of the correlator presented above, or an exponential decline of correlations in agreement with the observed non-scaling of the correlator. To account for the suspected additional finite-size corrections present in the estimate (24)
TABLE II: Decay exponent $a$ resp. wandering exponent $\omega$ related by Eq. (13) for the ensemble of regular dynamical triangulations as estimated by various scaling methods. In each row, the directly measured value is printed in bold face.

\begin{tabular}{lll}
\hline \hline Method & \multicolumn{1}{c}{$a$} & \multicolumn{1}{c}{$\omega$} \\
\hline Eq. (18) & $\mathbf{1 . 9 1 9 ( 7 6 )}$ & $0.7601(95)$ \\
Eq. (20) & $\mathbf{2 . 0 9 ( 1 3 )}$ & $0.739(16)$ \\
Eq. (25) & $2.021(78)$ & $\mathbf{0 . 7 4 7 3 ( 9 8 )}$ \\
\hline average & $1.987(76)$ & $0.7516(95)$ \\
\hline \hline
\end{tabular}

for dynamical triangulations, an additional FSS analysis is conducted by performing the analysis described above for the series of different graph sizes under consideration, ranging from $N=1000$ to $N=500000$. The resulting FSS plot is depicted in Fig. 9. We expect a scaling approach of the following form,

$$
\omega(N)=\omega_{\infty}+A N^{-\theta},
$$

with an a priori unknown correction exponent $\theta$. A fit of this form to the data yields:

$$
\begin{aligned}
\omega_{\infty} & =0.7473(98), \\
A & =-0.73(37), \\
\theta & =0.264(70), \\
Q & =0.28,
\end{aligned}
$$

Note that the correction exponent is close to $1 / d_{h}=1 / 4$, which would correspond to an analytic scaling correction. Fixing $\theta=1 / 4$, we find the very similar result $\omega_{\infty}=0.7493(23)$. For the Poissonian Voronoï-Delaunay triangulations, on the other hand, we find only pretty small variations of the estimates $\omega(N)$ with the graph size, which are about of the same size as the statistical fluctuations. Especially, for the largest two or three graph sizes, there is no visible drift between the estimates, such that we can safely take the result (24) for $N=500000$ triangles as our final estimate for the wandering exponent there.

Finally, we also checked for the influence of the number of disorder replicas on the results for the wandering exponent from the averaged fluctuations. For the Delaunay triangulations of size $N=500000$, using 50 resp. 10 instead of 100 realizations yields estimates of $\omega=0.50046(73)$ resp. $\omega=0.49818(469)$, in very good agreement with the result for the full number of replicas. In the case of the dynamical triangulations graphs, reducing the number of replicas to 50 resp. 10 and performing the fits (25) with the correction exponent $\theta$ fixed at $1 / 4$, we arrive at $\omega=0.7481(31)$ resp. $\omega=0.7590(72)$. With an unconstrained correction exponent $\theta$, we find the data with only 10 replica not precise enough to reliably apply the non-linear fit procedure. In Table II we collect the 
final results for the decay exponent $a$ resp. the wandering exponent $\omega$ for the case of dynamical triangulations from the various methods applied, using Eq. (13) with $d_{h}=4$ to compute $\omega$ from the directly estimated decay exponent $a$ or vice versa.

\section{CONCLUSIONS}

We have considered the applicability of a relevance criterion of the Harris-Luck type to the problem of coupling lattice models of statistical mechanics to the ensembles of Poissonian Voronoï-Delaunay random lattices and dynamical triangulations resp. planar, "fat" $\phi^{3}$ Feynman diagrams. Following Luck's extension of Harris' original argument to the case of systems with aperiodicity, a relevance criterion is formulated for the case of random graphs with connectivity disorder, i.e., a random distribution of co-ordination numbers. Depending on a characteristic of the spatial correlations of the disorder degrees of freedom termed the wandering exponent $\omega$, the threshold of relevance for a model with specific-heat exponent $\alpha$ predicted by the relevance criterion is shifted from Harris' value $\alpha_{c}=0$ to somewhere in the interval $-\infty<\alpha_{c} \leq 1$, depending on the value of $\omega$.

To determine the values of the wandering exponent for the considered ensembles of random graphs, we have employed a detailed series of scaling studies. First, we directly considered the behavior of the connected two-point correlation function of the co-ordination numbers of the vertices of the triangulations. For the Poissonian random lattices we find the correlations to decay very rapidly, and our analysis indicates that this decline is faster than any power of the distance. For dynamical triangulations, on the other hand, we find much stronger correlations, exhibiting a power-law decay within the scaling window. Due to the large fractal dimension of these lattices, however, we find quite strong corrections to the leading scaling behavior to be present. Taking the correction terms carefully into account, we are able to determine the decay exponent $a$ consistently with different methods, namely a direct analysis of the correlator, a finite-size scaling study of the correlation function at fixed distances and a scaling collapse of the data on a universal scaling function. Via Eq. (13) this yields then an estimate for the wandering exponent $\omega$. In addition to this analysis of the correlator, we investigate the scaling of the averaged fluctuations in spherical patches of increasing size, an expression which directly occurs in the definition of the wandering exponent. For the Voronoï-Delaunay random lattices we find an estimate for the wandering exponent consistent with a value of $\omega=1 / 2$ to high precision, which is in turn in agreement with the conjecture of correlations decaying faster than any power of the distance. Thus from Eq. (10), for Delaunay triangulations the presented relevance criterion reduces to the Harris criterion $\alpha_{c}=0$, such that disorder of this type should be relevant for any model with positive specific-heat exponent. For dynamical triangulations, on the other hand, a combination of the analyses presented allows us to conjecture ${ }^{86}$ that the correlator decays with an exponent $a=2$, leading to a value of the wandering exponent of $\omega=3 / 4$. In view of the relevance criterion (10), this leads to a relevance threshold as small as $\alpha_{c}=-2$. As a consequence, the class of dynamical triangulations graphs should be a relevant perturbation for all known ordered models of statistical mechanics.

There has been a number of numerical and analytical investigations of the effect of disorder from the classes of graphs considered here on the two-dimensional Potts model. For the case of a quenched ensemble of dynamical triangulations, a series of Monte Carlo simulations of the $q=2,3,4$ Potts model with $\alpha=0,1 / 3,2 / 3$, respectively, showed a change of their critical exponents, indicating a shift to new universality classes ${ }^{44,45,72}$. Additionally, it appears that the first-order transitions of the Potts models with $q>4$ get softened to second-order ones as the model is coupled to a quenched ensemble of dynamical triangulations ${ }^{44}$. For the case of percolation, corresponding to the $q \rightarrow 1$ limit of the Potts model, for which due to its non-interacting character quenched and annealed averages coincide, an exact solution can be obtained by use of matrix model methods ${ }^{73}$. This solution as well as numerical simulations for this model ${ }^{74}$, which has $\alpha=-2 / 3$, also yield changed critical exponents, in agreement with the relevance threshold obtained here. For the case of Poissonian random lattices, simulations of the Ising or $q=2$ Potts model, corresponding to the marginal case $\alpha=0$, yielded unchanged Onsager values for the critical exponents ${ }^{39-41,75}$; similar results where obtained for percolation ${ }^{76}$, both results being in agreement with the relevance threshold $\alpha_{c}=0$. On the other hand, for the $q=3,4$ Potts models with $\alpha>0$, Poissonian random lattices should be a relevant perturbation. However, an exploratory Monte Carlo study ${ }^{77}$ as well as preliminary results from a high-precision series of Monte Carlo simulations of the authors ${ }^{78}$ for the three-states Potts model do not show any change of universal behavior, in contradiction with the relevance threshold proposed here. It remains an open question to be answered, e.g., by further high-precision analyses of the $q=3$ case as well as the larger- $\alpha q=4$ Potts and Baxter-Wu $\mathrm{Wu}^{79}$ models, whether these findings are merely the effect of a crossover to new universal behavior occurring only for extremely large lattice sizes or whether there is possibly some physical reason for an argument of the Harris-Luck type not being applicable to the case of spin models coupled to Poissonian random lattices. For this purpose, a careful analysis of the counter-examples found in Refs. 61-68 might be instructive.

\section{Acknowledgments}

This work was partially supported by the EC research network HPRN-CT-1999-00161 "Discrete Random Ge- 
ometries: from solid state physics to quantum gravity" and by the German-Israel-Foundation (GIF) under contract No. I-653-181.14/1999. M.W. acknowledges support by the DFG through the Graduiertenkolleg "Quantenfeldtheorie".

\section{APPENDIX A: NEAREST-NEIGHBOR CORRELATIONS FOR QUANTUM GRAVITY GRAPHS}

This Appendix is devoted to a short derivation of the result for the co-ordination number correlator at distance one, $G_{q q}(1)$, for the ensemble of quantum gravity graphs mentioned in Sec. IV A. Godrèche et al. ${ }^{54}$ consider topological correlations in the thermodynamic limit of the ensemble of (regular) dynamical triangulations via a generating-function technique. This allows them to compute the probability distribution $Q_{l n}$ of finding an edge connecting a vertex with co-ordination number $l$ with a vertex with co-ordination number $n$ by means of a series expansion. This quantity is related to the probability of finding an $l$-vertex in the neighborhood of an $n$-vertex, which is of interest here, as follows:

$$
P_{l n}=\frac{1}{Z} \frac{36}{l n} Q_{l n}
$$

where

$$
Z=\sum_{l, n} \frac{36}{l n} Q_{l n}
$$

Then, the distance-1 correlator can be expressed as

$$
G_{q q}(1)=\sum_{l, n} P_{l n}(6-l)(6-n)=\frac{36}{Z}(1-12 S+Z),
$$

where $S=\sum_{l, n} Q_{l n} / l$. Integrating the generating function of $Q_{l n}$,

$$
Q(x, y)=\sum_{l, n} Q_{l n} x^{l} y^{n}
$$

one arrives at:

$$
\begin{aligned}
P(y) & =\int_{0}^{1} \mathrm{~d} x \frac{Q(x, y)-Q(0, y)}{x}=\sum_{l, n} \frac{Q_{l n}}{l} y^{n}, \\
Z & =36 \int_{0}^{1} \mathrm{~d} y \frac{P(y)-P(0)}{y}=36 \sum_{l, n} \frac{Q_{l n}}{l n} .
\end{aligned}
$$

The first integral can be performed exactly, yielding $S=$ $P(1)=1 / 6$. The double integral in the second line of Eq. (A5) could not be evaluated in closed form. Instead, the series expansion of $Q(x, y)$ used in Ref. 54, performed up to orders $l \leq 50$ and $n \leq 100$ (and additionally exploiting the symmetry property $Q_{l n}=Q_{n l}$ ) yields $Z \approx 0.96697$. Hence, we arrive at

$$
G_{q q}(1)=36 \frac{Z-1}{Z} \approx-1.2295 .
$$

\section{APPENDIX B: FINITE-SIZE SCALING OF THE CORRELATOR}

In this Appendix we give a short justification of the finite-size scaling (FSS) method used in Sec. IV A to determine the scaling exponent of the two-point correlator of co-ordination numbers and the correction terms taken into account. We consider making a real-space renormalization transformation with a re-scaling factor $b$ (see, e.g., Refs. 1,80,81). After $n$ iterations of the re-scaling, the two-point correlation function of some operator $\phi$ under consideration can be written as

$$
G_{\phi \phi}\left(R ; t, \frac{1}{N}\right)=b^{-2 x_{\phi} n} G_{\phi \phi}\left[\frac{R}{b^{n}} ; t b^{n y_{t}}, \frac{1}{N} b^{n d_{h}}\right],
$$

where $x_{\phi}$ denotes the scaling dimension of the operator $\phi$ and $y_{t}$ the temperature-related renormalization-group eigenvalue. Stopping the scale transformation at an iteration such that $N^{-1} b^{n d_{h}} \equiv K$, i.e., $b^{n}=(K N)^{1 / d_{h}} \equiv$ $\left(N / N_{0}\right)^{1 / d_{h}}$, we arrive at

$$
G_{\phi \phi}\left(R ; 0, \frac{1}{N}\right)=\left(\frac{N}{N_{0}}\right)^{-\frac{2 x_{\phi}}{d_{h}}} \hat{W}\left[\frac{R}{\left(N / N_{0}\right)^{\frac{1}{d_{h}}}}\right],
$$

introducing a universal scaling function $\hat{W}$. Therefore, if $R$ is scaled linearly with $N^{1 / d_{h}}$, e.g., $R^{*}=N^{1 / d_{h}} / 2$, the correlation function scales as

$$
G_{\phi \phi}\left(R^{*} ; 0, \frac{1}{N}\right) \sim N^{-\frac{2 x_{\phi}}{d_{h}}} .
$$

Now, instead of doing FSS, consider the finite-size "field" as a scaling correction and stop the re-scaling at $R / b^{n}=K$,

$G_{\phi \phi}\left(R ; t, \frac{1}{N}\right)=\left(\frac{R}{R_{0}}\right)^{-2 x_{\phi}} F\left[t\left(\frac{R}{R_{0}}\right)^{y_{t}}, \frac{1}{N}\left(\frac{R}{R_{0}}\right)^{d_{h}}\right]$,

where $F$ is another scaling function. Hence, at criticality one has

$$
\begin{aligned}
G_{\phi \phi}\left(R ; 0, \frac{1}{N}\right) & =\left(\frac{R}{R_{0}}\right)^{-2 x_{\phi}} \hat{F}\left[\frac{1}{N}\left(\frac{R}{R_{0}}\right)^{d_{h}}\right] \\
& \approx\left(\frac{R}{R_{0}}\right)^{-2 x_{\phi}}\left(\hat{F}(0)+\frac{1}{N}\left(\frac{R}{R_{0}}\right)^{d_{h}} \hat{F}^{\prime}(0)\right),
\end{aligned}
$$

which should be a reasonable approximation as long as $R \ll N^{1 / d_{h}}$. 
* Electronic address: janke@itp.uni-leipzig.de

$\dagger$ Electronic address: weigel@itp.uni-leipzig.de

1 J. L. Cardy, Scaling and Renormalization in Statistical Physics (Cambridge University Press, Cambridge, 1996).

2 A. P. Young, ed., Spin Glasses and Random Fields (World Scientific, Singapore, 1997).

3 M. Mézard, G. Parisi, and M. A. Virasoro, Spin Glass Theory and Beyond (World Scientific, Singapore, 1987).

${ }^{4}$ K. H. Fischer and J. A. Hertz, Spin Glasses (University Press, Cambridge, 1991).

${ }^{5}$ K. Binder and A. P. Young, Rev. Mod. Phys. 58, 801 (1986).

6 R. J. Elliott, B. R. Heap, D. J. Morgan, and G. S. Rushbrooke, Phys. Rev. Lett. 5, 366 (1960).

7 B. M. McCoy and T. T. Wu, Phys. Rev. Lett. 21, 549 (1968).

8 A. B. Harris, J. Phys. C 7, 1671 (1974).

${ }^{9}$ B. N. Shalaev, Phys. Rep. 237, 129 (1994).

10 B. Berche and C. Chatelain, in Order, Disorder And Criticality, edited by Y. Holovatch (World Scientific, Singapore, 2004), p. 147

11 J. T. Chayes, L. Chayes, D. S. Fisher, and T. Spencer, Phys. Rev. Lett. 57, 2999 (1986).

12 J. T. Chayes, L. Chayes, D. S. Fisher, and T. Spencer, Commun. Math. Phys. 120, 501 (1989).

13 A. W. W. Ludwig, Nucl. Phys. B 285, 97 (1987).

14 A. W. W. Ludwig and J. L. Cardy, Nucl. Phys. B 285, 687 (1987).

15 A. W. W. Ludwig, Nucl. Phys. B 330, 639 (1990).

16 J. M. Luck, Europhys. Lett. 24, 359 (1993).

17 J. L. Cardy, Physica A 263, 215 (1999).

18 H. G. Ballesteros, L. A. Fernández, V. Martín-Mayor, A. Muñoz Sudupe, G. Parisi, and J. J. Ruiz-Lorenzo, Phys. Rev. B 58, 2740 (1998).

19 P.-E. Berche, C. Chatelain, B. Berche, and W. Janke, Comput. Phys. Commun. 147, 427 (2002).

20 M. Hellmund and W. Janke, Comput. Phys. Commun. 147, 435 (2002).

21 J. L. Cardy and J. L. Jacobsen, Phys. Rev. Lett. 79, 4063 (1997).

22 J. L. Jacobsen, Phys. Rev. E 61, R6060 (2000).

23 C. Chatelain, B. Berche, and L. N. Shchur, J. Phys. A 34, 9593 (2001).

${ }^{24}$ Y. Imry and M. Wortis, Phys. Rev. B 19, 3580 (1979).

25 M. Aizenman and J. Wehr, Phys. Rev. Lett. 62, 2503 (1989).

${ }^{26}$ K. Hui and A. N. Berker, Phys. Rev. Lett. 62, 2507 (1989).

${ }^{27}$ K. Hui and A. N. Berker, Phys. Rev. Lett. 63, 2433 (1989).

${ }^{28}$ H. G. Ballesteros, L. A. Fernández, V. Martín-Mayor, A. Muñoz Sudupe, G. Parisi, and J. J. Ruiz-Lorenzo, Phys. Rev. B 61, 3215 (2000).

29 C. Chatelain, B. Berche, W. Janke, and P. E. Berche, Phys. Rev. E 64, 036120 (2001).

${ }^{30}$ M. Hellmund and W. Janke, Phys. Rev. E 67, 026118 (2003).

31 A. Weinrib and B. I. Halperin, Phys. Rev. B 27, 413 (1983).

32 V. V. Prudnikov and A. A. Fedorenko, J. Phys. A 32, L399 (1999).

33 P. T. Muzy, A. P. Vieira, and S. R. Salinas, Phys. Rev. E 65, 046120 (2002).
${ }^{34}$ V. Blavatska, C. von Ferber, and Y. Holovatch, Phys. Rev. B 67, 061103 (2003).

35 T. Vojta, J. Phys. A 36, 10291 (2003).

36 A. Okabe, B. Boots, K. Sugihara, and S. N. Chiu, Spatial Tessallations - Concepts and Applications of Voronoi Diagrams (Wiley, New York, 2000).

37 G. Schliecker, Adv. Phys. 51, 1319 (2002).

38 J. Ambjørn, B. Durhuus, and T. Jonsson, Quantum Geometry - A Statistical Field Theory Approach (Cambridge University Press, Cambridge, 1997).

39 D. Espriu, M. Gross, P. E. L. Rakow, and J. Wheater, Nucl. Phys. B 265, 92 (1986).

40 W. Janke, M. Katoot, and R. Villanova, Phys. Lett. B 315, 412 (1993).

41 W. Janke, M. Katoot, and R. Villanova, Phys. Rev. B 49, 9644 (1994).

42 W. Janke and R. Villanova, Phys. Lett. A 209, 179 (1995).

43 K. N. Anagnostopoulos, P. Bialas, and G. Thorleifsson, J. Stat. Phys. 94, 321 (1999).

44 W. Janke and D. A. Johnston, Nucl. Phys. B 578, 681 (2000).

45 W. Janke and D. A. Johnston, J. Phys. A 33, 2653 (2000).

${ }^{46}$ W. Janke and R. Villanova, Phys. Rev. B 66, 134208 (2002).

47 R. Albert and R.-L. Barabási, Rev. Mod. Phys. 74, 47 (2002).

${ }^{48}$ F. Iglói and L. Turban, Phys. Rev. E 66, 036140 (2002).

49 J. M. Drouffe and C. Itzykson, Nucl. Phys. B 235, 45 (1984).

50 D. V. Boulatov, V. A. Kazakov, I. K. Kostov, and A. A. Migdal, Nucl. Phys. B 275, 641 (1986).

51 M. E. Agishtein and A. A. Migdal, Nucl. Phys. B 350, 690 (1991).

52 E. Brézin, C. Itzykson, G. Parisi, and J.-B. Zuber, Commun. Math. Phys. 59, 35 (1978).

53 M. L. Mehta, Random Matrices (Academic Press, San Diego, 1996), 2nd ed.

${ }^{54}$ C. Godrèche, I. Kostov, and I. Yekutieli, Phys. Rev. Lett. 69, 2674 (1992).

55 S. Jain and S. D. Mathur, Phys. Lett. B 286, 239 (1992).

56 H. Kawai, N. Kawamoto, T. Mogami, and Y. Watabiki, Phys. Lett. B 306, 19 (1993).

57 Y. Watabiki, Nucl. Phys. B 441, 119 (1995).

58 E. Domany, Phys. Rev. B 12, 1038 (1975).

59 W. Kinzel and E. Domany, Phys. Rev. B 23, 3421 (1981).

60 D. Andelman and A. N. Berker, Phys. Rev. B 29, 2630 (1984).

61 B. Derrida, H. Dickinson, and J. Yeomans, J. Phys. A 18, L53 (1985).

62 S. Mukherji and S. M. Bhattacharjee, Phys. Rev. E 52, 1930 (1995).

63 A. C. N. de Magalhaes, S. R. Salinas, and C. Tsallis, J. Phys. A 31, L567 (1998).

64 T. A. S. Haddad, S. T. R. Pinho, and S. R. Salinas, Phys. Rev. E 61, 3330 (2000).

65 A. Efrat, J. Phys. A 63, 066112 (2001).

66 F. Pázmándi, R. T. Scalettar, and G. T. Zimányi, Phys. Rev. Lett. 79, 5130 (1997).

67 A. Aharony, A. B. Harris, and S. Wiseman, Phys. Rev. Lett. 81, 252 (1998).

68 A. L. Korzhenevskii, H. O. Heuer, and K. Herrmanns, J. 
Phys. A 31, 927 (1998).

69 J. Ambjørn, P. Bialas, and J. Jurkiewicz, JHEP 9902, 005 (1999).

70 B. Efron, The Jackknife, the Bootstrap and Other Resampling Plans (Society for Industrial and Applied Mathematics [SIAM], Philadelphia, 1982).

71 B. Efron and R. J. Tibshirani, An Introduction to the Bootstrap (Chapman and Hall, Boca Raton, 1998).

72 A. Wernecke, W. Janke, and D. A. Johnston (unpublished).

73 V. A. Kazakov, Mod. Phys. Lett. A 4, 1691 (1989).

${ }^{74}$ G. Harris, Nucl. Phys. B 418, 278 (1994).

75 F. W. S. Lima, J. E. Moreira, J. S. Andrade Jr., and U. M. S. Costa, Physica A 283, 100 (2000).

76 H.-P. Hsu, S. C. Lin, and C.-K. Hu, Phys. Rev. E 64, 016127 (2001).

77 F. W. S. Lima, U. M. S. Costa, M. P. Almeida, and J. S. Andrade, Eur. Phys. J. B 17, 111 (2000).

78 M. Weigel and W. Janke (unpublished).

79 R. J. Baxter, Exactly Solved Models in Statistical Mechanics (Academic Press, London, 1982).

${ }^{80}$ M. E. Barber, in Phase Transitions and Critical Phenom- ena, edited by C. Domb and J. L. Lebowitz (Academic Press, New York, 1983), vol. 8, p. 146.

81 M. Henkel, Conformal Invariance and Critical Phenomena (Springer, Berlin/Heidelberg/New York, 1999).

82 All distances on the graphs considered in this paper are to be understood as the unique number of links in the shortest path of links connecting two vertices.

83 An implicit assumption in applying such scaling arguments is that the ensemble of considered random graphs behaves as a system of statistical mechanics at a critical point. From the dynamical triangulations approach to quantum gravity it is known that this is indeed the case, see, e.g., Ref. 38

84 Note that due to the correlations between values of $G_{q q}(R)$ for different distances $R$, the absolute values of $\chi^{2}$ resp. $Q$ are not immediately meaningful; relative changes, however, are.

85 The number of vertices of a spherical triangulation consisting of $N$ triangles is $N_{0}=2+N / 2$.

86 Note that all critical exponents of the dynamical triangulations model, which could be calculated exactly, where found to be rational numbers, see, e.g., Ref. 38 . 